\title{
Factors affecting germination of ascospores in Aspergillus fumigatus
}

\begin{abstract}
Background and objective: Aspergillus fumigatus is the most common airborne fungal pathogen of humans. It is an opportunistic human pathogen in immunocompromised hosts. Following the recent discovery of the sexual reproductive cycle ascospores of this fungus were available and their germination was studied in the present study. This study aimed to study factors influencing germination of ascospores of $A$. fumigatus.

Methods: A total of 12 environmental $A$. fumigatus were chosen in both mating type, examined for sexual reproduction by crossing complementary mating type, following removing of cleistothecia, preparation of ascospore suspensions in $0.05 \%$ Tween 20 , then heat treated to kill any remaining conidia.
\end{abstract}

Results: Ascospore germination of greater than $75 \%$ occurred at $28{ }^{\circ} \mathrm{C}$ on a wide variety of mycological media, $0.5 \%$ (ACM) was chosen for use in subsequent studies. Ascospores did not require a heat treatment prior to germination; however, heat treatment of ascospores at $75{ }^{\circ} \mathrm{C}$ for 1 hour previous to incubation enhanced germination to $89 \%$ and also served to inactivate contaminating conidia. Ascospores also exhibited a psychrotolerance following exposure at $-80{ }^{\circ} \mathrm{C}$ for 96 hours. Inoculum concentration did not have a significant effect on germination rates between the range of $0.125 \times 10^{6}$ to $4 \times 10^{6}$ ascospores $\mathrm{ml}^{-1} \quad(P>0.05)$.

Conclusion: This study demonstrated that ascospore germination of greater than $75 \%$ occurred at $28{ }^{\circ} \mathrm{C}$ on a wide variety of mycological media. Ascospores of $A$. fumigatus are thermotolerant also exhibited a psychrotolerance whilst at the same time retaining viable sexual ascospores, to assess percentage germination a drop of Lactophenol Cotton Blue was added.

Keywords: Ascosopre germination; A. fumigates; Factors.

\section{Introduction}

A. fumigatus is a saprotrophic fungus that is involved in environmental carbon and nitrogen recycling is a ubiquitous filamentous fungus in the environment. It is abundant in soils and decomposing organic materials. It is essential that $A$. fumigatus survive and flourishes in nutrient limited environments, ${ }^{1}$ and the most widespread airborne human pathogen, accountable for approximately $90 \%$ of all invasive aspergillosis (IA) cases. $^{2}$ Spores in $A$. fumigatus can be produced by two different forms of cellular reproduction, asexual reproduction (produce conidia) or sexual reproduction (produce ascospore).
Sexual reproduction involves the production of eight ascospores normally produced in each ascus. These asci form within an ascocarp with a closed structure called cleistothecium. The species has a heterothallic breeding system, isolates of complementary mating types are necessary for sex to take place. ${ }^{3}$ Sexual reproduction may bring about increased genetic variation, due to recombination of genes from the parents that can occur by crossing over, gene conversion, or independent segregation of centromeres, permitting a species to react to a change in the environment and evolve. ${ }^{4}$ Germination consequently forms a first and critical stage

* Department of Basic Sciences, College of Dentistry, Hawler Medical University, Erbil, Iraq. 
of the infectious process in the invasive pathogenic lifestyle of $A$. fumigatus; three important steps can be distinguished during spore germination, namely activation, isotropic growth, and polarized growth. ${ }^{5}$ Many aspergilli produce ascospores with relatively thick cell walls, which might again explain their superior ability to withstand adverse environmental conditions such as temperature and desiccation compared to conidia. ${ }^{6}$ In certain Aspergillus species, the ascospores are constitutive dormancy and may require a special activation treatment involving conditions, such as high or low temperature or light or addition of a diverse of chemical agents and vitamins. ${ }^{7}$ The thermotolerance ascospores of Aspergillus fischeri, which is strongly related to A. fumigatus, require an external trigger such as heat for germination $A$. fischeri was identified as a contaminant of canned strawberries; ascospores had survived heating of between $77^{\circ} \mathrm{C}$ and $90{ }^{\circ} \mathrm{C}$ for 30 minutes. ${ }^{8}$ Furthermore a requirement for exposure of spores to high temperature to ensure germination is especially common among saprotrophic ascomycetes, e.g., ascospores of Neurospora, Byssochlamys, Talaromyces, and Neosartorya species often require exposure to high temperatures between $60-90^{\circ} \mathrm{C}$ to trigger germination. ${ }^{9}$ Such strong shock causing damage and changes in structure of the cell wall of these spores, which then allows germination. $^{10}$ By contrast most spores of basidiomycete species, especially plant pathogens, are stimulated to germinate by exposure to low temperatures of 5 to $10^{\circ}$ C. ${ }^{11}$ It has been suggested that a medium contains a higher level of soluble ingredients might enhance the heat activation of ascospores of Eurotium species given the fact that this genus is xerophilic. $^{12}$ In addition, self-inhibitory substances secreted by spores in competition for space and nutrients had a significant effect.It was established that at higher inoculum concentration of conidia in $A$. fumigatus such as those greater than $1 \times 10^{6}$ conidia $\mathrm{ml}^{-1}$ produced lower levels of germination. ${ }^{13}$ This study aimed to detect the factors affecting ascospore germination of sexual ascospores of $A$. fumigatus is highly significant in assessing whether the sexual ascospores exhibited differential resistance to environmental stress, which might be important for the evolutionary maintenance and testing the pathogenicity of ascospores to establish their potential as opportunistic human pathogens.

\section{Methods \\ Collection and isolation of $\boldsymbol{A}$. fumigatus isolates}

The 12 isolates isolated from environmental are in storage in the School of Biology, University of Nottingham, the UK as $10 \%$ glycerol stocks under liquid nitrogen.

\section{Ascospore germination}

In order to determine the best conditions to study ascospore germination, a series of preliminary experiments were conducted in order to identify suitable mycological media, Effect of surfactant (Tween-20 detergent), ascospore concentration, and thermotolerance of ascospores, Psychrotolerance of ascospores. Also, studies were made to identify the optimum heat shock to break the dormancy and activate the ascospore germination whilst at the same time allowing the elimination of temperature sensitive conidia

\section{Removal of cleistothecia.}

Using an Olympus SZH10 Stereo dissecting microscope and cleistothecia were picked off from the mycelium and transferred to a water agar plate. Cleistothecia were gently rolled across the water agar surface in order to keep the cleistothecium intact, using a sterilised needle tip to remove any adhering conidia. Preparation of ascospore suspensions. Mature cleistothecia were picked off from surrounding hyphae using a flame sterilised needle, observed under a Nikon-SMZ-2B dissecting microscope, taking care to avoid conidia where possible. 5-10 cleistothecia were transferred to a $4 \%(\mathrm{w} / \mathrm{v})$ water agar plate 
and gently rolled across the agar surface using a sterilised needle tip to remove any adhering conidia, Next, $20-50 \mu \mathrm{l}$ of $\mathrm{pH}$ 6 sterile Tween $20(\mathrm{BDH})(0.05 \%)$ was pipetted into a sterile $1.5 \mathrm{ml}$ Eppendorf tube and cleistothecia ruptured against the side of the tube in this small droplet, This was made up to a final volume of $500 \mu \mathrm{l}$ in $\mathrm{pH} \quad 6$ sterile Tween $20 \quad(0.05 \%)$ vortex-mixing for 1 minute to release the ascospores. The concentration of the resulting ascospore suspension was determined using a Haemocytometer and were diluted as appropriate. For heat treatment of ascospore suspensions the Eppendorf tubes containing acospore suspension were inserted into holes on a polyfoam tray which was suspended over a $75^{\circ} \mathrm{C}$ water bath unpublished. ${ }^{14}$

\section{Inoculation of Petri dishes}

A template and a fine tipped red permanent marker were used to outline three defined circular areas on the underside of each of two Petri dishes, typically $0.5 \%$ Aspergillus Complete Medium (ACM). The circular areas were $2.0 \mathrm{~cm}$ in diameter and $1.5 \mathrm{~cm}$ apart to ensure that spores were acting independently. The spore suspension was pipetted into each circle in a laminar air flow cabinet and spread inoculated using an inoculation loop, incubation at $28{ }^{\circ} \mathrm{C}$ for 18 hours.

\section{Assessing germination}

The American Phytopathological Society's (1943) definition of germination as being "the presence of a visible germ tube." Germination was stopped by adding a drop of magenta Lactophenol Cotton Blue Stain and placing a coverslip onto each circle.
Clumps of more than six ascospores were not counted. Germinated and ungerminated ascospores were counted, 100 ascospores were counted per circle, and as there were six replicates, 600 ascospores were counted per treatment.

Germination of ascospores of Aspergillus fumigatus on different media

In order to select an optimal medium for ascospore germination, eleven mycological media types and Agar No. 3 as control were inoculated with six replicates for each media. Spore concentration was calculated as $0.55 \times 10^{6}$ ascospores $\mathrm{ml}^{-1}$. The media used in this study were: $0.5 \%$ Aspergillus Complete Medium (ACM), Agar No. 3 $\left(\mathrm{H}_{2} \mathrm{O}\right)$, Corn Meal Agar (CMA, Oxoid), Czapek Dox Agar (CZPD, Oxoid), King B Agar (KINGB), Malt Extract Agar (MEA, Oxoid), Morinda Medium (NAX), Mung Bean Agar (MUNGB), Nutrient Agar (NA, Oxoid), Potato Dextrose Agar (PDA, Oxoid), Potato Dextrose Agar (PDA, Oxoid) $+1 \%$ Mycological Peptone, and Sabouraud-2\% (SAB).

Thermotolerance of ascospores of Aspergillus fumigatus

To assess the effect of heat exposure on ascospore germination rate, Eppendorf tubes containing ascospore suspensions were placed in a water bath at $75{ }^{\circ} \mathrm{C}$ and $90{ }^{\circ} \mathrm{C}$ for periods up to 120 minutes (Table 1). Spore concentrations were calculated as $0.312 \times 10^{6}$ ascospores $\mathrm{ml}^{-1}$. Two Petri dishes were each inoculated in triplicate per time incubated at $28{ }^{\circ} \mathrm{C}$ for $18 \mathrm{~h}$.

Table 1: Exposure temperature and time of ascospore suspensions to heat treatment.

\begin{tabular}{lcccccccccccc}
\hline Temperature $\left({ }^{\circ} \mathbf{C}\right)$ & Control & \multicolumn{10}{c}{ Time (Minutes) } \\
\hline 75 & 0 & 5 & 10 & 15 & 20 & 25 & 30 & 40 & 50 & 60 & 90 & 120 \\
90 & 0 & 10 & 20 & 40 & 60 & 90 & 120 \\
9
\end{tabular}


Effect of surfactant (Tween-20 detergent) on germination of ascospores of $\mathrm{A}$. fumigatus

To determine whether $0.05 \%$ Tween-20 detergent (BHD Chemicals, UK) used to make up acospore suspensions had an effect on ascospore germination rate. Suspensions were set up as with or without $0.05 \%$ Tween-20 detergent where water was used as a control and heat treated at $75^{\circ} \mathrm{C}$ for 60 minutes Spore concentration was calculated as $1.18 \times 10^{6}$ ascospores $\mathrm{ml}^{-1}$. Two Petri dishes each were inoculated in triplicate and incubated at incubation at $28^{\circ} \mathrm{C}$ for 18 hours.

Psychrotolerance of ascospores of Aspergillus fumigatus

To ascertain whether ascospores survive and germinate following exposure to temperatures up to $-80{ }^{\circ} \mathrm{C}$ for up to 96 hours, ascospore suspensions were made up and subjected to three different temperatures for a range of time periods (Table 2). A control of 0 minute exposure and a heat treatment at $75^{\circ} \mathrm{C}$ for 60 minutes in a water bath were also set up. $0^{\circ} \mathrm{C}$ was maintained by filling a stainless steel canister with ice into which Eppendorf tubes were inserted, this was then left in a $5^{\circ} \mathrm{C}$ cold room, while -20 and $-80^{\circ} \mathrm{C}$ fridge freezers facilitated at these temperatures.

Effect of ascospore concentration ('population effect') on germination of ascospores of Aspergillus fumigatus

To assess whether the level of ascospore concentration exerts an effect on the germination rate of $A$. fumigatus, six different concentrations were made up starting with a master concentration of $4 \times 10^{6}$ ascospores $\mathrm{ml}^{-1}$ which was heat treated at $75^{\circ} \mathrm{C}$ for 60 minutes, concentrations from $0.125 \times 10^{6}, 0.25 \times 10^{6}$, $0.5 \times 10^{6} 1 \times 10,{ }^{6} 2 \times 10^{6}, 4 \times 10^{6}$ ascospores per $\mathrm{ml}$ incubation at $28^{\circ} \mathrm{C}$ for 18 hours.

Statistical Analysis: The statistics software (SPSS, version 21) for Macintosh (Mac OS X version 10.6.8) was used for data analysis.

Table 2: Exposure temperature and time of ascospore suspensions to cold treatment.

\begin{tabular}{lllllllll}
\hline Temperature $\left({ }^{\circ} \mathrm{C}\right)$ & \multicolumn{3}{c}{ Time (Minutes) } & \multicolumn{2}{c}{ Time (Hours) } \\
\hline-80 & 1 & 2 & 3 & 4 & 5 & 60 & 24 & 96 \\
$\mathbf{- 2 0}$ & 1 & 2 & 3 & 4 & 5 & 60 & 24 & 96 \\
$\mathbf{0}$ & 1 & 2 & 3 & 4 & 5 & 60 & 24 & 96 \\
Control & 0 & & & & & & & \\
\hline
\end{tabular}
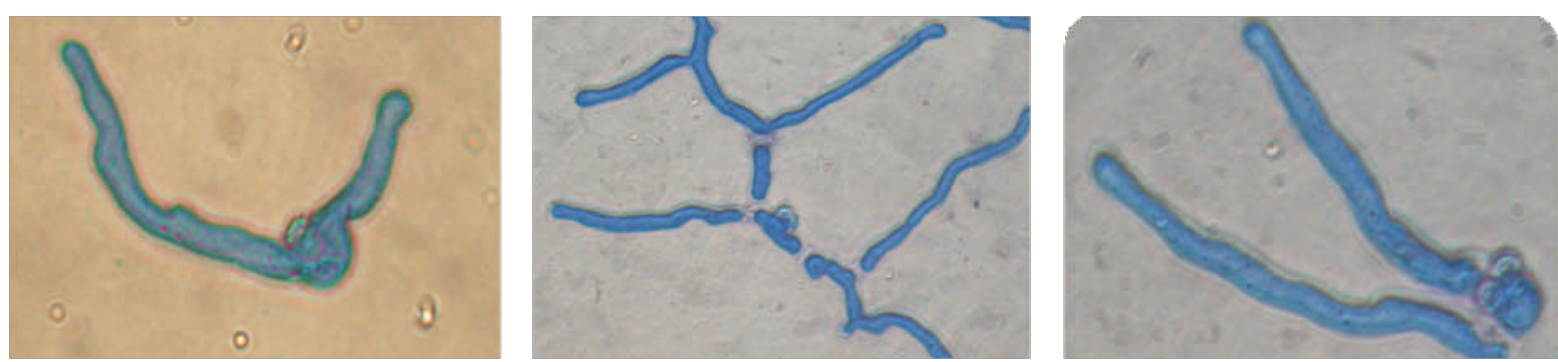

Figure 1: Images of ascospore germination stained by lactophenol cotton blue. 


\section{Results}

Germination of ascospores of Aspergillus fumigatus on different media

Following incubation at $28{ }^{\circ} \mathrm{C}$ for 18 hours, mean germination of ascospores was greater than $75 \%$ on the broad range of media. Czapek Dox Agar (CZPD) was recorded as having the lowest ascospore percentage germination of $75.33 \pm 2.94 \%$ ( $n=600$; mean \pm s.e.m.) while the control, Agar No. $3\left(\mathrm{H}_{2} \mathrm{O}\right)$ had a germination rate of $80.16 \pm 2.05 \%$ (Table 3 ). Analysis of the collated results demonstrated that 0.5\% Apergillus Complete Medium (ACM) was the optimal solid medium for ascospore germination for use in subsequent experiments in this project. Ascospore percentage germination rate of $89.5 \pm 1.38 \%$ was measured for $0.5 \%$ ACM, although Potato Dextrose Agar+ (PDA+) and Morinda Medium NAX) had values of $91.0 \pm 1.78 \%$ and $92.83 \pm 2.37 \%$ respectively. Additionally, $0.5 \%$ ACM was a defined medium and was specific to Aspergillus species requirements containing optimal nutrient quantities. Furthermore, there was no significant difference between the mean values of PDA+ and NAX $(P>0.05)$. PDA+ and NAX.

Table 3: Mean percentage germination (\%) of an ascospore suspension inoculated onto various media and incubated at $28{ }^{\circ} \mathrm{C}$ for 18 hours with six replicates for each media $(n=600)$.

Media

Mean percentage germination (\%)

Czapek Dox (CZPD)

King B (KINGB)

Agar No. $3\left(\mathrm{H}_{2} \mathrm{O}\right)$

Malt Extract Agar (MEA)

Corn Meal Agar (CMA)

Potato Dextrose Agar (PDA)

Mung Bean (MUNGB

Nutrient Agar (NA)

Sabouraud (SAB)

0.5\% Aspergillus Complete Medium (ACM.5)

Potato Dextrose Agar $+1 \%$ Mycological Peptone (PDA+)
75.33

75.5

80.16

80

81

81

82.4

84

85

89.5

91

92.83 
Thermotolerance of ascospores of Aspergillus fumigatus

The thermotolerance of ascospores exposed to $75^{\circ} \mathrm{C}$ for $0-120$ minutes (Figure 2) demonstrated that ascospores without any pre-exposure had the lowest ascospore percentage germination of $9.16 \pm 0.79 \%$. At 5 minutes percentage germination of $74.5 \pm 4.89 \%$ was recorded, while the highest mean germination of
$91.16 \pm 2.85 \%$ was measured at 90 minutes. Germination of ascospores without any pre-exposure was found to be highly statistically significant to the other time intervals of 5-120 minutes which were heat treated $(P<0.001)$. Germination was not recorded at any time interval following exposure to heat treatment of $90^{\circ} \mathrm{C}$ for 0-120 minutes.

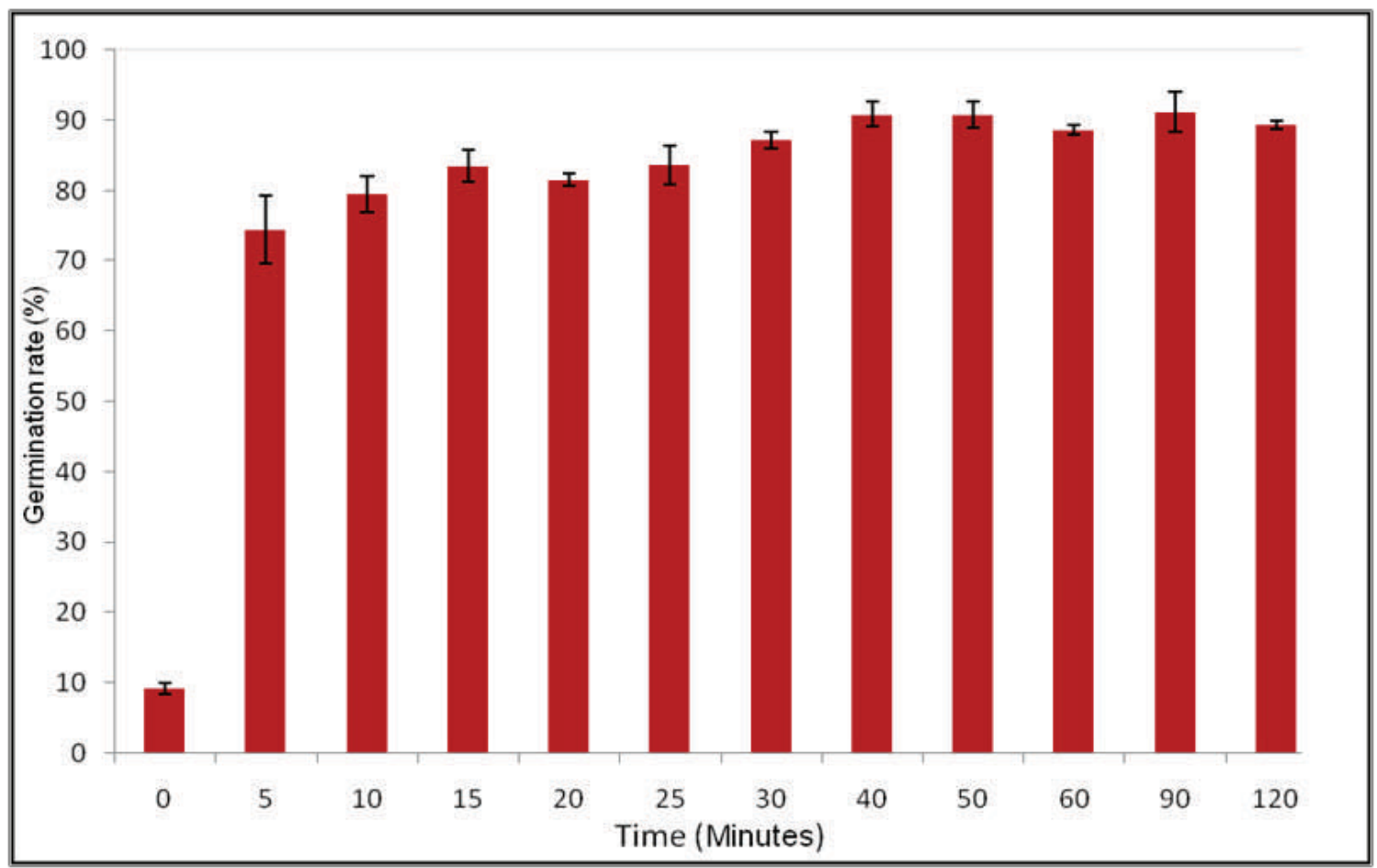

Figure 2: Mean percentage germination (\%) of an ascospore suspension exposed to $75{ }^{\circ} \mathrm{C}$ heat treatment for $0-120$ minutes and incubated at $28{ }^{\circ} \mathrm{C}$ for 18 hours with six replicates per time interval $(n=600)$. Data of plot symbols labeled with the same letter are not significantly different from each other $(P>0.05)$. Error bars represent standard errors of the mean. 
Effect of surfactant (Tween-20 detergent) on germination of ascospores of Aspergillus fumigatus

There was no statistically significant difference between mean percentage germination presence or absence of the Tween-20 detergent $(P=0.29)$. However, germination of ascospores at times intervals of 1 to 3 minutes (Figure 3 ) showed significantly higher mean germination levels in suspensions where tween was present $(P<0.05)$. There was no statistically significant difference between mean germination for 4 to 60 minutes either in the presence or absence of tween $(P>0.05)$.

Psychrotolerance of ascospores of Aspergillus fumigatus

(Table: 4) demonstrates that ascospores exhibited psychrotolerance as they germinated after exposure to cold treatment of up to $-80^{\circ} \mathrm{C}$ for up to 96 hours when incubated at $28^{\circ} \mathrm{C}$ for 18 hours.

Table 4: Presence of ascospore germination following exposure to cold temperatures of $-80,-20,0{ }^{\circ} \mathrm{C}$, exposure to $+75^{\circ} \mathrm{C}$ for 60 minutes, a control without treatment and time per treatment. The Petri dishes were inoculated with six replicates per time interval $(n=600)$.

\begin{tabular}{lccc}
\hline Temperatur $\left({ }^{\circ} \mathrm{C}\right)$ & Time (Minutes) & Time (Hours) & Ascospore Germination \\
\hline 0 & $0-60$ & $24-96$ & + \\
-20 & $0-60$ & $24-96$ & + \\
-80 & $0-60$ & $24-96$ & + \\
+75 & 60 & - & + \\
Control & 0 & - & + \\
\hline
\end{tabular}

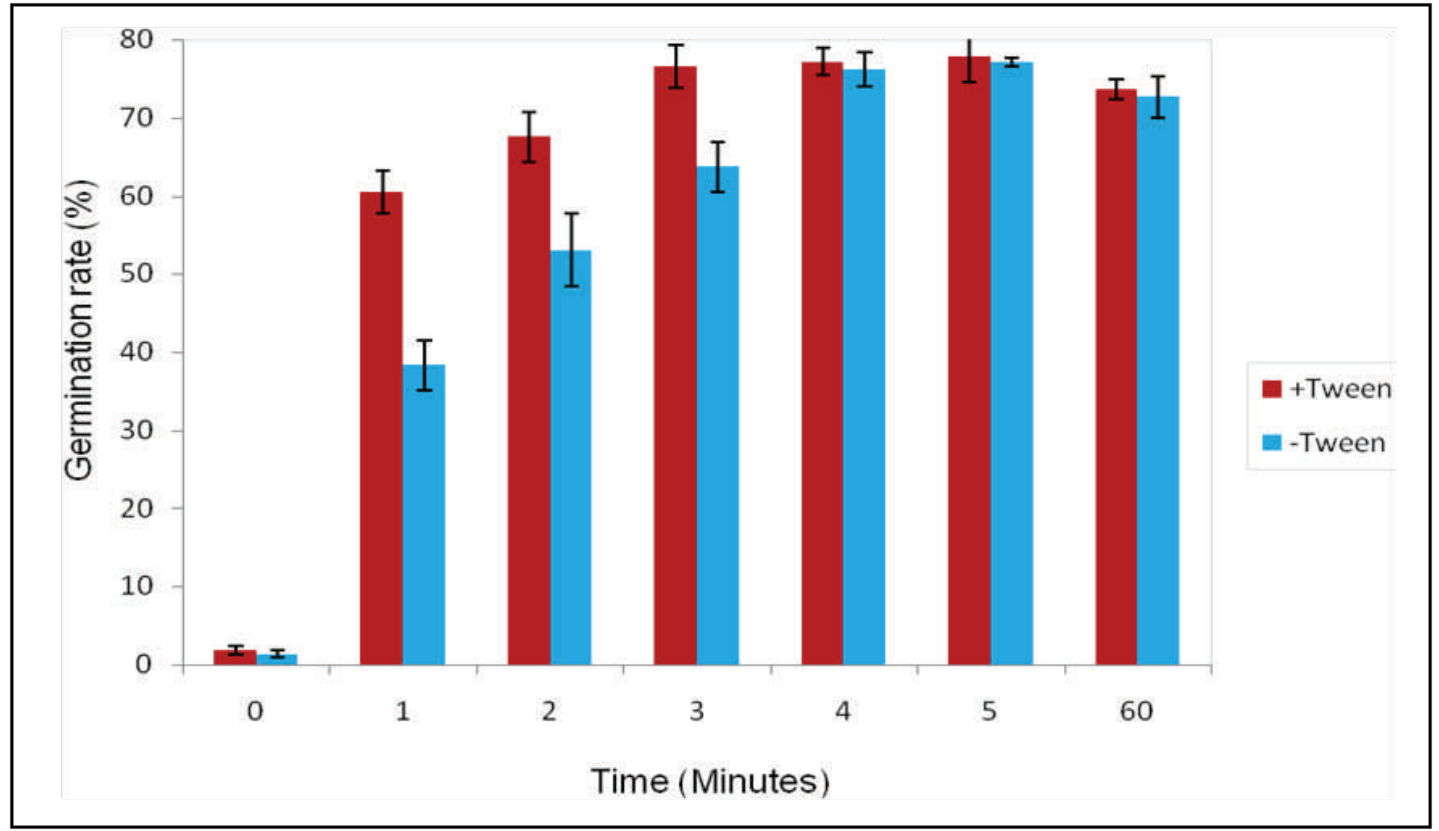

Figure 3: Mean percentage germination (\%) of ascospore suspensions with or without $0.05 \%$ Tween-20 detergent where water was used as a control, suspensions were exposed to $75{ }^{\circ} \mathrm{C}$ heat treatment for $0-60$ minutes and incubated at $28{ }^{\circ} \mathrm{C}$ for $18 \mathrm{~h}$ with six replicates per time interval $(n=600)$. Error bars represent standard errors of the mean. 
Effect of ascospore concentration ('population effect') on germination of ascospores of Aspergillus fumigatus

Figure 4 revealed that there was no statistically significant difference in the mean percentage of germination between the inoculum concentration range of $0.125 \times 10^{6}$ to $4 \times 10^{6}$ ascospores $\mathrm{ml}^{-1}$ $(P>0.05)$.

\section{Discussion}

O'Gorman et al. $^{3}$ recently discovered that $A$. fumigatus possesses a sexual reproductive cycle, which leads to the production of cleistothecia and ascospores and the teleomorph Neosartorya fumigata. This study demonstrated that ascospores of Aspergillus fumigatus are thermotolerant. Investigations established that ascospores were stimulated to germinate following 5 minutes of exposure to a heat treatment of $75{ }^{\circ} \mathrm{C}$, germination occurred on $0.5 \% \mathrm{ACM}$ incubation at $28{ }^{\circ} \mathrm{C}$ for $18 \mathrm{~h}$. Greater than $75 \%$ ascospore germination was recorded on the wide variety of mycological media including the water control; this indicated that ascospores were undemanding of nutrient requirements. This suggested that the ascospore contained all the essential nutrients that are needed for the early stage of germination as reserves. As A. fumigatus is a saprotrophic fungus and its airborne conidia are abundant in soils and compost heaps it is essential that A. fumigatus survive and flourishes in nutrient limited environments. This capacity to perceive carbon and nitrogen and the adaptability of the organism to utilise different compounds enables $A$. fumigatus to compete against other microflora in compost and to operate as an efficient opportunistic pathogen of immuno-suppressed hosts. ${ }^{15}$ It was realized that ascospores were thermotolerant and exposure to $75^{\circ} \mathrm{C}$ stimulated germination following 5 minutes. Germination of ascospores without any pre-exposure was found to be highly statistically significant in comparison to the other treatments at different intervals that had been subjected to heating $(P<0.001)$. Given that ascospores had germinated without heating, this verified that A. fumigatus ascospores do not require a heat shock prior to germination. However, ascospores displayed a germination rate of greater than $70 \%$ at 5 minutes and greater

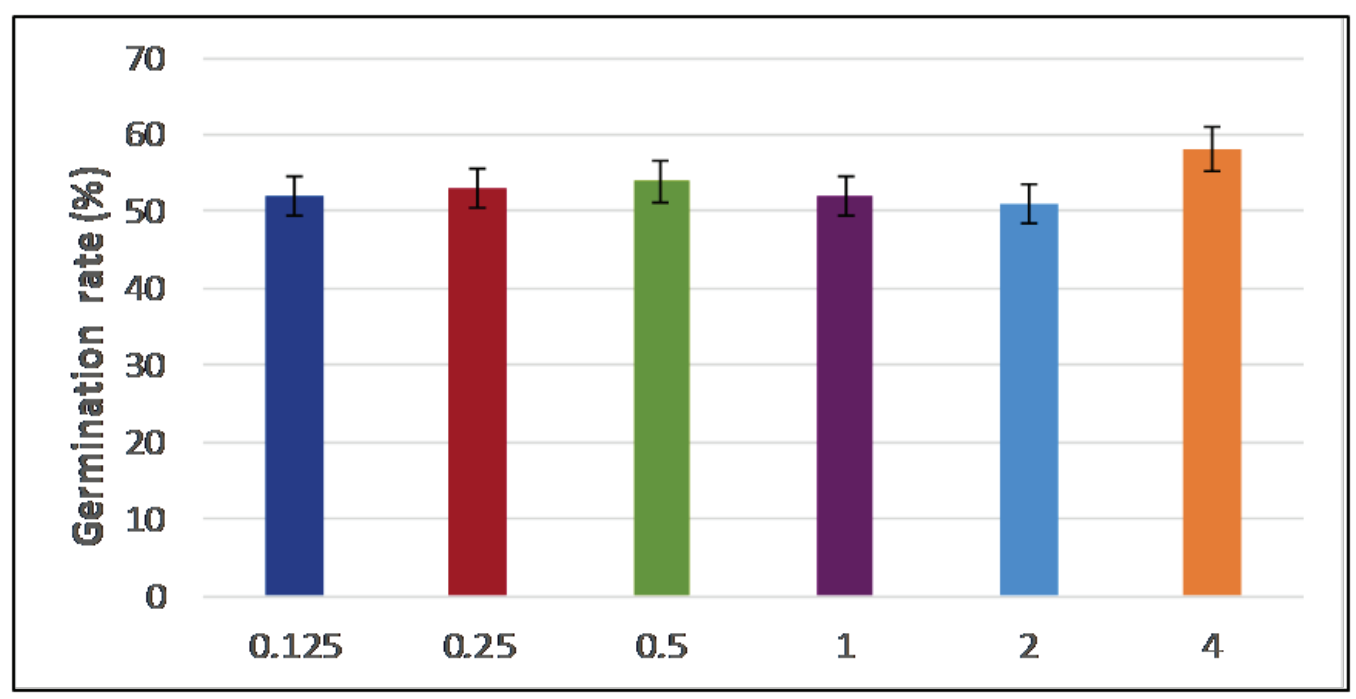

Figure 4: Mean percentage of germination (\%) of ascospore suspensions ranging from $0.125 \times 10^{6}$ to $4 \times 10^{6}$ ascospores $\mathrm{ml}^{-1}$ and incubated at $28{ }^{\circ} \mathrm{C}$ for $18 \mathrm{~h}$ with six replicates per concentration $(n=600)$. Data of plot symbols labelled with the same letter are not significantly different from each other $(P>0.05)$. Error bars represent standard errors of the mean. 
than $86 \%$ at 120 minutes following heat exposure. It was evident from these values that ascospores were capable of not only surviving but demonstrated high germination levels following exposure to $75^{\circ} \mathrm{C}$ for up to 2 hours. ${ }^{5} A$. fumigatus is strongly related to $A$. fischeri, the conidial stages of these fungi are nearly identical ${ }^{8}$ studied thermotolerance of both conidia and ascospores of $A$. fischeri var. glaber. Their findings demonstrated an apparent contrast between ascospore germination of $A$. fumigatus and $A$. fischeri. Since dormant ascospores of $A$. fischeri germinated only after an external heat shock of $70^{\circ} \mathrm{C}$ and above. On the contrary, the heating inactivated any contaminating conidia present in $A$. fumigatus suspensions, and $A$. fischeri conidia remained ungerminated following heat exposure. Dormant ascospores of Talaromyces macrosporus, Neurospora and Byssochlamys also need a heat trigger to break dormancy and to activate germination. Several studies have investigated heat resistance of these spores that spoil food products such as fruit juice. ${ }^{16}$ The ability of $T$. macrosporus ascospores to germinate following heat exposure was due to the presence of trehalose protecting cell components as glucose was released from the cell. ${ }^{17}$ There was no statistically significant difference between mean percentage germination presence or absence of the Tween-20 detergent $(P=0.29)$. However.germination of ascospores at times intervals of 1 to 3 minutes showed significantly higher mean germination levels in suspensions where tween was present $(P<0.05)$. The results of Gomez-Lopez et al. $^{18}$ confirm the importance of the concentration of surfactant Tween- 20 to use on inoculum preparation for antifungal susceptibility testing of Aspergillus spp. Significantly higher MICs was associated with higher concentrations of Tween- 20. Moreover, surfactant Tween -80, at $0.02 \%$ concentration was most effective for enhancement of $\alpha$-amylase production in culture media by Aspergillus niger. $^{19}$
A. fumigatus ascospores also proved to be psychrotolerant as they germinated following exposure to cold treatments of $0^{\circ} \mathrm{C},-20^{\circ} \mathrm{C}$ and $-80{ }^{\circ} \mathrm{C}$ for up to 96 hours. Psychrotolerant fungi can grow at low temperatures of $5^{\circ} \mathrm{C}$ or lower but favour more moderate temperatures. The physiological mechanisms conferring cold tolerance in fungi are comprised of increases in intracellular trehalose and polyol concentrations and unsaturated membrane lipids. Additionally, secretion of antifreeze proteins and enzymes active at low temperatures are also involved. Many cold-tolerant fungi grow in common environments, such as Penicillium and Cladosporium species growing at $4{ }^{\circ} \mathrm{C}$ and $0{ }^{\circ} \mathrm{C}$ respectively on food in fridges. ${ }^{20}$ It was verified that the germination rate in the inoculum concentration range of $0.125 \times 10^{6}$ to $4 \times 10^{6}$ ascospores $\mathrm{ml}^{-1}$ did not differ significantly $(P>0.05)$. This suggested that there was no population effect on ascospore germination whereby an increase in space due to a lower density did not improve germination rates. In contrast, a study on germination of A. fumigatus conidia by Manavathu et al. ${ }^{21}$ proposed that high conidial densities such as those greater than $1 \times 10^{6}$ conidia $\mathrm{ml}^{-1}$ produce lower levels of germination which they suggested was a consequence of a population effect.

\section{Conclusion}

This study demonstrated that ascospore germination of greater than $75 \%$ occurred at $28{ }^{\circ} \mathrm{C}$ on a wide variety of mycological media. Ascospores of $A$. fumigatus are thermotolerant also exhibited a psychrotolerance whilst at the same time retaining viable sexual ascospores, to assess percentage germination a drop of Lactophenol Cotton Blue was added.

\section{Competing interests}

The author declares no competing interests. 


\section{References}

1. Latgé JP.Aspergillus fumigatus and Aspergillosis. Clin Microbiol Rev 1999; 12:310-50.

2. Denning DW. Invasive Aspergillosis. Clin Infect Dis 1998; 26:781-803.

3. O'Gorman CM, Fuller HT, Dyer PS. Discovery of a sexual cycle in the opportunistic fungal pathogen Aspergillus fumigatus. Nature 2009; 457:471-4.

4. Barton $\mathrm{NH}$, Charlesworth B. Why sex and recombination? Science 1998; 281:1986-90.

5. Mouchacca J. Heat tolerant fungi and applied research: Addition to the previously treated group of strictly thermotolerant species. World J Microbiol Biotechnol 2007; 23:1755-70.

6. Dijksterhuis J, Nijsse J, Hoekstra FA, Golovina EA. High viscosity and anisotropy characterise the cytoplasm of fungal dormant stress-resistant spores. Eukaryot Cell 2007; 6:157-70.

7. Coluccio $A E$, Rodriguez RK, Kernan MJ, Neiman AM. The yeast spore wall enables spores to survive passage through the digestive tract of Drosophila. Plos One 2008; 3: e2873.

8. McEvoy IJ, Stuart MR. Temperature tolerance of Aspergillus fischeri var. glaber in canned strawberries. Ir J Agric Res 1970; 9:59-67.

9. Beuchat L. Extraordinary heat resistance of Talaromyces flavus and Neosartorya fischeri ascospores in fruit products. J Food Sci 1986; 51: 1506_10.

10. Dijksterhuis J, Teunissen P. Dormant ascospores of Talaromyces macrosporus are activated to germinate after treatment with ultra high pressure. J Appl Microbiol 2003; 96:162-9.

11. Leach CM. Regulation of perithecium development and maturation in Pleospora herbarum by light and tempreture. Trans $\mathrm{Br}$ mycol Soc 1971; 57:295-315.

12. Splittstoesser D, Lammers J, Downing D, Churey J. Heat resistance of Eurotium herbariorum, a xerophilic mold. J Food Sci 1989; 54:683_5.

13. Araujo R, Rodrigues AG. Variability of germinative potential among pathogenic species of Aspergillus. J. Clin. Microbiol 2004; 42:43357.

14. Swilaiman SS. Sexual Potential and Population Biology of Fungal Aspergillus and Penicillium species, PhD thesis. University of Nottingham; 2013.

15. Rhodes JC. Aspergillus fumigatus: Growth and virulence. Med Myco 2006; 44:S77-81.

16. Dijksterhuis J, Teunissen PG. Dormant ascospores of Talaromyces macrosporus are activated to germinate after treatment with ultra high pressure. J Appl Microbiol 2004; 96:162-9.

17. Dijksterhuis J, van Driel KG, Sanders MG, Molenaar D, Houbraken JA, Samson RA, et al. Trehalose degradation and glucose efflux precede cell ejection during germination of heat-resistant ascospores of Talaromyces macrospores. Arch Microbiol 2002; 178:1-7.
18. Gomez-Lopez A, Aberkane A, Petrikkou E, Mellado E, Rodriguez-Tudela J, Cuenca-Estrella M. Analysis of the Influence of Tween Concentration, Inoculum Size, Assay Medium, and Reading Time on Susceptibility Testing of Aspergillus spp. J clinic Microbiol 2005; 43:12515.

19. Gupta A, Gautam N, Modi D. Optimization of a-amylase production from free and immobilized cells of Aspergillus nige. E3 J Biotechnol Pharmacol Res 2010; 1:001-8.

20. Deacon JW. Fungal Biology. $4^{\text {th }}$ ed. Oxford, UK: Blackwell Publishing; 2006.

21. Manavathu EK, Cutright J, Chandrasekar $\mathrm{PH}$. Comparative study of susceptibilities of germinated and ungerminated conidia of Aspergillus fumigatus to various antifungal agents. J Clin Microbiol 1999; 37:858-61. 